\title{
Online estimation of rollator user condition using spatiotemporal gait parameters
}

\author{
Joaquin Ballesteros $^{1}$, Cristina Urdiales ${ }^{1}$, Antonio B. Martinez ${ }^{2}$ and Marina Tirado ${ }^{3}$
}

\begin{abstract}
Assistance to people during rehabilitation has to be adapted to their needs. Too little help can lead to frustration and stress in the user; an excess of help may lead to low participation and loss of residual skills. Robotic rollators may adapt assistance. The main challenge to cope with this issue is to estimate how much help is needed on the fly, because it depends not only on the person condition, but also on the specific situation that they are negotiating. Clinical scales provide a global condition based estimation, but no local estimator based on punctual needs. Condition also changes in time, so clinical scales need to be recalculated again and again. In this paper we propose a novel approach to estimate users' condition in a continuous way via a robotic rollator. Our work focuses on predicting the value of the well known Tinetti Mobility test from spatiotemporal gait parameters obtained from our platform while users walk. This prediction provides continuous insight on the condition of the user and could be used to modify the amount of help provided. The proposed method has been validated with 19 volunteers at a local hospital that use a rollator for rehabilitation. All volunteers presented some physical or mental disabilities. Our results sucessfully show a high correlation of spatiotemporal gait parameters with Tinetti Mobility test gait $(R 2=0.7)$ and Tinetti Mobility test balance $(R 2=0.6)$.
\end{abstract}

\section{INTRODUCTION}

Population is getting older. The elderly are projected to grow by 56 per cent until 2030 [1]. In addition, a high percent of population aged 16 and over declared a severe disability [2]. There are not enough care giving professionals to cover these needs, so in extreme many of these people require institutionalization. Assistive robots may improve the autonomy and the quality of life of people with dependency [3] to avoid these problems. Medical specialists recommend to use a variety of assistive devices depending on the user requirements [4]. In this paper we focus on smart rollators (Fig. 1) which are a useful support for activities of daily living (ADL).

Walking assistance and fall prevention have been extensively investigated in the field of smart rollators [5], [6], [7], [8], [9]. However, only a few works [10], [11] propose to adjust the amount of assistance. Adjustment is critical for rehabilitation purposes. Unnecessary assistance provokes a

*This work has been partially supported by the MINECO (Proj. TEC2011-29106, Proj. TEC2014-56256- C2-1-P), Hospital Regional Universitario of Malaga and Fondazione Santa Lucia of Rome.

${ }^{1}$ Joaquin Ballesteros, and Cristina Urdiales is with Department of Electronic Technology, University of Malaga, Malaga, Spain jballesteros@uma.es acurdiales@uma.es

${ }^{3}$ Antonio B. Martinez is with the Department of Automatic Control, Polytechnic University of Catalonia, Barcelona, Spain antonio.b.martinez@upc.edu

${ }^{3}$ Marina Tirado is with UGC Rehabilitacion in Hospital Regional, Malaga, Spain mtiradoreyes@gmail.com

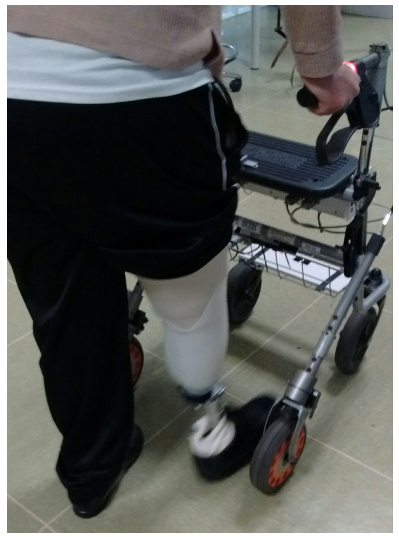

Fig. 1. The user 8 walks with i-Walker platform

decrease of physical and mental activities, increasing the risk of a disuse syndrome [12]. Too little help may lead to failure, frustration and stress.

Some works on help adjustment define different states depending on user posture [13]. When unusual states are detected, they reactively change the amount of help. However, they do not analyze how much help different users may need if they are in the same state. Another approach takes into account the user condition as provided by medical staff feedback [10]. They manually adjust some rollator parameters in order to modify the amount of help beforehand. These solutions obviously depend on clinicians' feedback and can not adapt to condition changes or special situations unless a new clinical assessment is performed. [11] excludes clinical intervention by using the center of rotation to evaluate the user condition. However, this parameter needs to be calibrated from a test without assistance. If its value changes in time, it needs to be recalibrated.

In this work we propose a novel approach to unsupervisedly estimate user condition using only spatiotemporal gait parameters obtained on the fly by the sensors in a robot rollator. We will correlate those parameters with the well known Tinetti Mobility Test [14] while users walk. Thus, we will isolate the most relevant parameters that provide the same information than Tinetti does, only these parameters can be unsupervisedly obtained any time while users walk. The main contributions of this work are: i) normalization and scaling of spatiotemporal gait parameters related to user condition; ii) obtaining a prediction of Tinetti Mobility Test using only spatiotemporal gait parameters; and iii) validation with a set of volunteers presenting a diversity of physical and/or cognitive disabilities. Our results have been successful 
in every case. We expect to use this estimation in the future to adapt the amount of help provided by the rollator to users in a continuous way.

\section{Methodology}

\section{A. The $i$-Walker platform and our gait analysis algorithm}

We use the smart rollator i-Walker [15]. It is a standard rollator frame with force sensors embedded in its handlebars and encoders in both wheels (Fig. 1).

In our previous work [16] we used i-Walker to obtain meaninful spatiotemporal gait parameters from different challenged users. Our results validated that the chosen set of spatiotemporal gait parameters is coherent with the user diagnosed condition. However, we still did not use the parameter set to estimate how much help each user required because we needed to combine those parameters into a single estimation. In this work, we obtain that estimation using only the handlebar force sensors and the wheels odometry. Unlike other solutions which include cameras or wearable sensors, estimation can be obtained anywhere, in everyday conditions and for extended periods of time.

The gait analysis algorithm presented in [16] returns 7 parameters (Table I). Some parameters as UserSupport or StepTime depend on body side. We obtain average and standard deviation of each parameter for a period of time. It total there are 18 features to be analyzed $\left\{P_{1}, P_{2}, \ldots, P_{n}\right\}$.

\section{B. Test population}

Testing assistive devices with users with disabilities is usually challenging, so many works in this area tend to test with healthy [7], [8], [17], [18] or simulated users [11]. Other works focus on specific groups of users, e.g. Parkinson [19] or stroke [20]. Our goal is to define a general estimation of rollator user condition. Therefore, we have chosen a set of volunteers as varied as possible at the Hospital Regional Universitario de Malaga and Fondazione Santa Lucia. Our volunteers had a variety of cognitive and/or physical disabilities (Table II). We imposed only two restrictions to our volunteers, they had to: i) be able to walk with the aid of a rollator; and ii) support some weight while walking on the rollator. With the second restriction, we discarded users who could walk without any aid.

Our tests include 19 volunteers: 13 women and 6 men. They are in average $67.47 \pm 8.86$ years old (range $46-80$ years). Table II shows their neurological and/or physical disabilities. We asked clinicians to provide the Tinetti scale results for every volunteer, so we could correlate gait parameters with Tinetti Balance $\left(T_{B A L}\right)$ and Gait $\left(T_{G A I T}\right)$. The table includes body size parameters for each volunteer, because parameters like stride length or user support depends on weight $\left(w_{0}\right)$ and leg length $\left(l_{0}\right)$ and they need to be normalized. The cognitive disabilities in our volunteers include Parkinson, dementia and intellectual disability. The physical disabilities include amputees and a variety of fractures. The Parkinson and Dementia patients were at the end of their rehabilitation process and their condition did not change.

\section{Mobility assessment and proposed test}

As commented, clinicians provided the Tinetti Mobility Test[14] for our volunteers. This test is divided into two steps: one to measure the balance $\left(T_{B A L}\right)$, and another to evaluate the gait function $\left(T_{G A I T}\right) . T_{B A L}$ is related with fall risk and equilibrium issues. $T_{G A I T}$ depends on how users walk. A healthy volunteer can obtain a maximum of 28 point: 16 point for a perfect balance and 12 point for a perfect gait function.

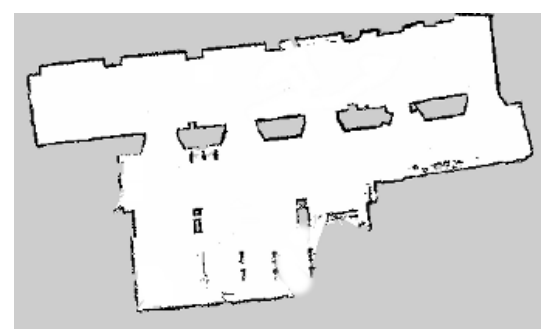

Fig. 2. Rehabilitation room map (approximately $128 \mathrm{~m}^{2}$ )

After finishing their Tinetti Mobility Test, volunteers walked freely around in the rehabilitation room (Fig. 2) for 3 minutes. Their paths needed to include steering right and left at least once smoothly and once sharply, plus a 10 meter straight walk. Further than that, the path changed during the sessions, depending on obstacles around and users' decisions. Other patients and the medical staff were allowed to walk freely in the same room during tests on purpose.

\section{PREDICTING GAIT AND BALANCE FROM SPATIOTEMPORAL GAIT PARAMETERS}

This section proposes a methodology to predict the gait and balance indicators proposed in Tinetti Mobility Assessment using only spatiotemporal gait parameters that can be extracted anytime on the fly from the onboard sensors of a smart rollator. The main advantage of this approach is that no medical intervention nor previous training is required to estimate how much help users may need depending on their gait and balance. Thus, this estimation can be obtained in a continuous, unsupervised way.

\section{A. Data preprocessing}

As commented, spatiotemporal gait parameters $\left\{P_{1}, P_{2}, \ldots, P_{n}\right\}$ are affected by the user's body size, e.g. tall people tend to walk with larger $S p L$ and lower $C A D$ than smaller people and $U r S$ obviously depends on the people weight. [21] proposes to scale all gait data using only leg length $l_{0}$ and user weight $w_{0}$.

Following the guidelines provided by [21], we divided our spatial parameters $(S d L, S p L)$ by the leg length $l_{0}$. Also, temporal parameters ( $S d T, S p T, C A D)$ have been divided by $\sqrt{\frac{l_{o}}{g}}$. This factor is obtained from the rate of acceleration with respect to space. $W V$ is a spatio-temporal parameter; therefore it has been divided by $l_{0}$ and multiplied by $\sqrt{\frac{l^{\prime} o}{g}}$. Also, force parameter $U s R$ has been divided by $\frac{1}{m_{0} g}$. After scaling, we have normalized parameters $\left\{P_{1}^{\prime}, P_{2}^{\prime}, \ldots, P_{n}^{\prime}\right\}$. 
TABLE I

SPATIOTEMPORAL GAIT PARAMETERS

\begin{tabular}{|c|c|c|c|c|}
\hline Name & Acronym & Description & Unit & Side \\
\hline Cadence & CAD & Number of steps per minute & $\frac{\text { step }}{\text { min }}$ & No \\
\hline Stride Time & SdT & Time difference between one step and the next in the same side & $s$ & $\begin{array}{c}\text { Left } \\
\text { Right }\end{array}$ \\
\hline Step Time & SpT & Time difference between one step and the next one & $s$ & $\begin{array}{c}\text { Left } \\
\text { Right }\end{array}$ \\
\hline Stride Length & SdL & Length difference between one step and the next in the same side & $m$ & $\begin{array}{l}\text { Left } \\
\text { Right }\end{array}$ \\
\hline Step Length & $\mathrm{SpL}$ & Length difference between one step and the next one & $m$ & $\begin{array}{l}\text { Left } \\
\text { Right }\end{array}$ \\
\hline Walking Velocity & WV & Number of steps per minute & $\frac{m}{s}$ & No \\
\hline User Support & $\mathrm{UrS}$ & Amount of weight that users support in rollators. Directly related with weight-bearing. & $N$ & $\begin{array}{l}\text { Left } \\
\text { Right }\end{array}$ \\
\hline
\end{tabular}

TABLE II

USERS CONDITION AND BODY SIZE

\begin{tabular}{|c|c|c|c|c|c|c|c|}
\hline Age & Gender & Neurological & Physical & $T_{B A L}$ & $T_{G A I T}$ & $w_{0}$ & $l_{0}$ \\
\hline \hline 65 & W & - & Periprosthetic femur fracture (Left) & $13-15$ & $10-12$ & $75 \mathrm{~kg}$ & $0.9 \mathrm{~m}$ \\
\hline 63 & W & - & Hip arthroplasty (Right) & $4-5$ & $2-6$ & $86.4 \mathrm{~kg}$ & $0.96 \mathrm{~m}$ \\
\hline 74 & $\mathrm{M}$ & - & Hip fracture, calcaneal and metacarpal (Left) & $14-16$ & $8-10$ & $57 \mathrm{~kg}$ & $0.85 \mathrm{~m}$ \\
\hline 68 & W & - & Left above-knee amputation. CREST syndrome & $7-12$ & $3-6$ & $56 \mathrm{~kg}$ & $0.95 \mathrm{~m}$ \\
\hline 58 & M & Intellectual disability & Right above-knee amputation & 9 & $7-8$ & $96.7 \mathrm{~kg}$ & $1.1 \mathrm{~m}$ \\
\hline 70 & M & Mild parkinson & - & 13 & 12 & $75 \mathrm{~kg}$ & $0.93 \mathrm{~m}$ \\
\hline 80 & W & Mild parkinson & - & 14 & 10 & $63 \mathrm{~kg}$ & $0.75 \mathrm{~m}$ \\
\hline 63 & M & Moderate parkinson & - & 8 & 12 & $91 \mathrm{~kg}$ & $0.97 \mathrm{~m}$ \\
\hline 78 & W & Moderate parkinson & - & 10 & 10 & $61 \mathrm{~kg}$ & $0.0 .81 \mathrm{~m}$ \\
\hline 67 & W & Moderate dementia & - & 11 & 10 & $55 \mathrm{~kg}$ & $0.85 \mathrm{~m}$ \\
\hline 74 & M & Mild parkinson & - & 13 & 12 & $73 \mathrm{~kg}$ & $0.83 \mathrm{~m}$ \\
\hline 71 & M & Isquemia (Left) & - & 12 & 6 & $72 \mathrm{~kg}$ & $0.99 \mathrm{~m}$ \\
\hline 71 & W & Mild parkinson & - & 13 & 11 & $68 \mathrm{~kg}$ & 0.75 \\
\hline 73 & W & Mild parkinson & - & 15 & 11 & $74 \mathrm{~kg}$ & $0.86 \mathrm{~m}$ \\
\hline 78 & W & Mild dementia & - & 15 & 11 & $74 \mathrm{~kg}$ & 0.86 \\
\hline 78 & W & Mild dementia & - & 16 & 12 & $52 \mathrm{~kg}$ & 0.79 \\
\hline
\end{tabular}

At this point, there is a high degree of correlation exist in the normalized parameters that we plan to use for prediction. For example, $W V$ depends on $S p L$ and $C A D$. Other parameters like $S d L$ and $S p L$ are also correlated. The Variance Inflation Factor (VIF) for our parameter after scaling is 883.43 in average (Fig. 3). It indicate a high multicollinearity in our data. Multicollinearity reportedly makes estimation very sensitive to slight changes[22].

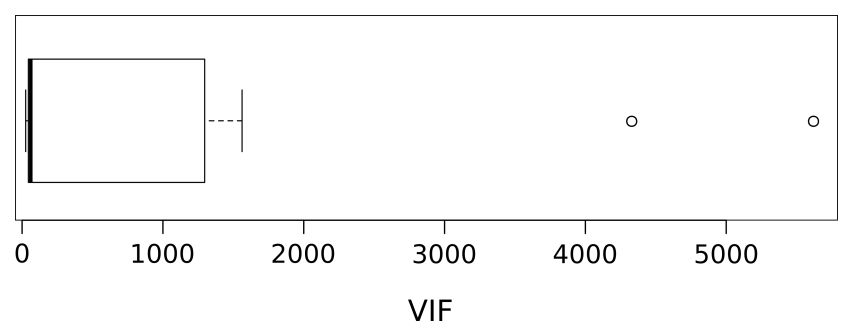

Fig. 3. VIF of gait parameters

\section{B. Multivariate regression: $P C R$ and PLS}

In order to reduce multicollinearity and check the correlation between our gait parameters and Tinnetti $T_{B A L}$ and $T_{G A I T}$, we have analyzed two potential multivariate regression methods: the principal component regression (PCR) [23] and partial least squares (PLS) [23]. These methods have been designed to deal with situations with correlated dependent variables and few samples. We use the pls $\mathrm{R}$ package implementation of both methods [24].

PCR is a multivariate regression that uses principal component analysis (PCA) to reduce multicolinearity before applying a linear regression to resulting data. PCA is a statistical procedure that uses an orthogonal transformation to maximize variability in analyzed data by returning a new set of variables called principal components. The (chosen) number of principal components $m$ is lower or equal to the original number of parameters $n$. If $m$ increases, then the multivariate variability increases too. In our case, PCA generates linear factors (loading) $\left\{\alpha_{1}^{C_{1}}, \alpha_{2}^{C_{1}}, \ldots, \alpha_{n}^{C_{m}}\right\}$ to transform the scaled spatiotemporal gait parameters for user $j\left\{P_{1}^{\prime j}, P_{2}^{\prime j}, \ldots, P_{n}^{\prime j}\right\}$ to the new PCA components (scores) $\left\{C_{1}^{j}, C_{2}^{j}, \ldots, C_{m}^{j}\right\}$. Equation 1 shows the transformation for component $C_{k}$ and user $j$.

$$
C_{k}^{j}=\alpha_{1}^{C_{k}} P_{1}^{\prime j}+\alpha_{2}^{C_{k}} P_{2}^{\prime j}+\alpha_{3}^{C_{k}} P_{3}^{\prime j} \ldots \alpha_{n}^{C_{k}} P_{n}^{\prime j}
$$

Afterwards, PCR does linear regression using the obtained components. A $m$ regression coefficients are obtained from the linear regression $\left\{\beta_{1}, \beta_{2}, \ldots, \beta_{m}\right\}$. Using these coefficients, the Tinetti Mobility factors for new user $i$ with scaled spatiotemporal gait parameters $\left\{P_{1}^{\prime i}, P_{2}^{\prime i}, \ldots, P_{n}^{\prime i}\right\}$ can 
be estimated using equation 2 .

$$
\begin{array}{r}
T_{\text {pred }}=\beta_{1}\left(\alpha_{1}^{C_{1}} P_{1}^{\prime i}+\alpha_{2}^{C_{1}} P_{2}^{\prime i}+\ldots+\alpha_{n}^{C_{1}} P_{n}^{\prime i}\right) \\
+\beta_{2}\left(\alpha_{1}^{C_{2}} P_{1}^{\prime i}+\alpha_{2}^{C_{2}} P_{2}^{\prime i}+\ldots+\alpha_{n}^{C_{2}} P_{n}^{\prime i}\right) \\
\ldots \\
+\beta_{m}\left(\alpha_{1}^{C_{m}} P_{1}^{\prime i}+\alpha_{2}^{C_{m}} P_{2}^{\prime i}+\ldots+\alpha_{n}^{C_{m}} P_{n}^{\prime i}\right)
\end{array}
$$

PLS works like PCR, but uses a different approach. PLS searches for directions that have high variance and correlation with respect to the dependent variable, in opposition to PCA which focus only on high variance [25]. PCR does not take into account correlation between the dependent and the independent variables and hence, it is usually suboptimal for prediction.

PLS also represents dependent variables using a new base with a number of components $m$. It also has a loading factor to transform scaled spatiotemporal gait parameters into the scores. Then, it uses these scores to find a linear regression model.

\section{Evaluating our models}

An important aspect when modeling a response variable is the cross validation $(\mathrm{CV})$ technique. CVs split observations in two: a part for learning purposes and another part for testing purposes. These techniques limit overfitting in the resulting models. In this paper, we apply a k-fold cross validation with $k=10$ to evaluate the regression with all users [26].

We are going to evaluate 2 models $\left(T_{B A L}\right.$ and $\left.T_{G A I T}\right)$ using 2 multivariate regression (PCR and PLS) with a number of components $1 \leq m \leq 18$. We are going to use the root mean squared prediction error (RMSEP) [27] and the multiple coefficient of determination (R2) [24] to select the best possible combination.

Let $T_{\text {pred }}$ be the prediction function for PCR or PLS, $L_{k}$ the test set in our k-folder cross validation, $\left\{C_{1}^{j}, C_{2}^{j}, \ldots, C_{m}^{j}\right\}$ the independent variables and $y_{j}$ the response variable. The RMSEP is calculated as:

$$
R M S E P=\sqrt{\sum_{k=1}^{K} \frac{1}{\left|L_{k}\right|} \sum_{j \in L_{k}}\left(T_{\text {pred }}\left(C_{1}^{j}, \ldots, C_{m}^{j}\right)-y_{j}\right)^{2}}
$$

Let $\bar{y}$ be the mean of the response variables. The $\mathrm{R} 2$ is defined as the ration between the variances of the fitted values and real values of the dependent variable:

$$
R 2=\sum_{k=1}^{K} \frac{1}{\left|L_{k}\right|} \frac{\sum_{j \in L_{k}}\left(T_{\text {pred }}\left(C_{1}^{j}, C_{2}^{j}, \ldots\right)-\bar{y}\right)}{\sum_{j \in L_{k}}\left(y_{j}-\bar{y}\right)}
$$

Low values of RMSEP represent a better model, i.e. fitted values are more similar to the real ones and it is not bounded. On the other hand, R2 is bounded between 0 and 1 and any value close to 1 means that the dependent variable can

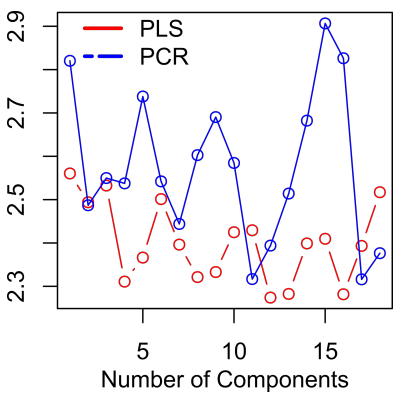

(a) RMSEP $T_{B A L}$

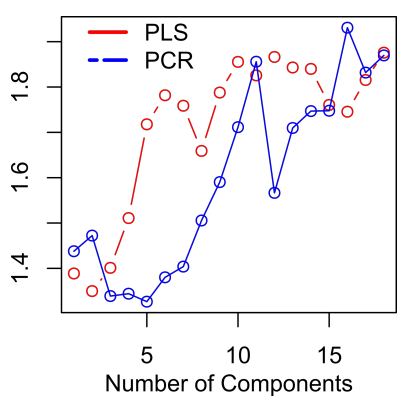

(c) RMSEP $T_{G A I T}$

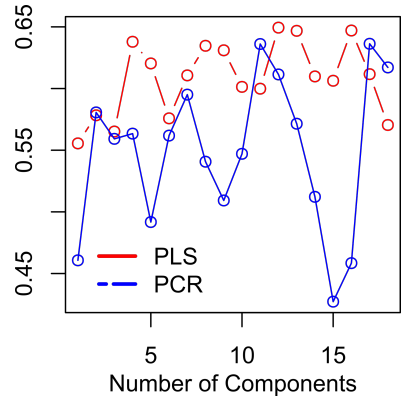

(b) $R 2 T_{B A L}$

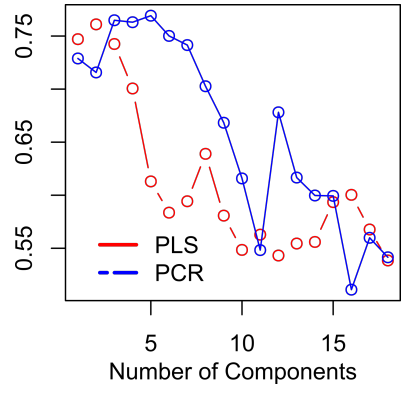

(d) $R 2 T_{G A I T}$
Fig. 4. R2 and RMSEP metrics for $T_{B A L}$ and $T_{G A I T}$ using PCR and PLS with all posible number of components

be predicted from the independent variables with a minimal error.

Fig.4 shows R2 and RMSEP metrics for $T_{B A L}$ and $T_{G A I T}$ using PCR and PLS with all possible numbers of components. The best combination is the one presenting the highest R2 value and the lowest RMSEP value.

For $T_{B A L}$, PLS provides better result. A low RMSEP value and high $\mathrm{R} 2$ value indicates that the depended variables can be predicted with an acceptable error. We observe four possible $m$ values in R2 priority (Fig. 4(b)): 4, 12, 13 and 16. In RMSEP we also observe the same four possible $m$ values (Fig. 4(a)). The error difference from selecting 4 components to selecting more is less than $1 \%$ in R2 and less than 0.0368 in RMSEP. These small improvements do not compensate an increase in the number of components, so we set for $m=4$.

For $T_{G A I T}$, PCR provides better results. We observe three possible $m$ values in R2 priority (Fig. 4(d)): 3, 4 and 5 . In RMSEP we observe the same three values (Fig. 4(c)). As in the previous case, the difference between selecting 3 components or more is less than $0.5 \%$ in $\mathrm{R} 2$ and less than 0.0123 in RMSEP. Therefore we set for $m=3$.

Fig. 5 shows the prediction using the selected number of components in both cases. $T_{G A I T}$ predictor outperforms $T_{B A L}$ predictor. It has a lower RMSEP value and a higher $\mathrm{R} 2$ value. This difference was expected. We are using spatiotemporal gait parameters to measure equilibrium and fall risk in $T_{B A L}$, wheras $T_{G A I T}$ focuses on the user gait, and hence, it can be predicted more accurately by using these parameters. 


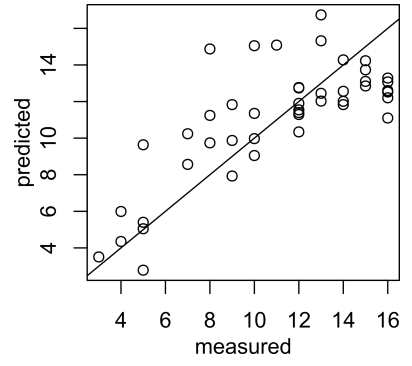

(a) $T_{B A L}$ prediction $m=4$

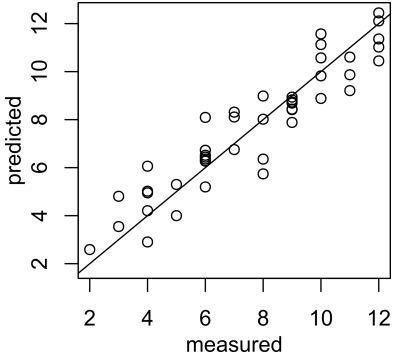

(b) $T_{G A I T}$ prediction $m=3$
Fig. 5. Cross validated prediction for Tinneti mobility test

\section{Extracting the model}

Once the number of components for $T_{G A I T}$ and $T_{B A L}$ is selected and results have been validated, our model for estimating a new dependent variables using the proposed spatiotemporal gait parameters can be obtained. Using the loading $\alpha_{n}^{C_{m}}$ and the regression coefficients $\beta_{m}$ values we can rearrange the equation 2 in order to obtain a compact equation:

$$
\begin{aligned}
T_{\text {pred }}= & P_{1}^{\prime i}\left(\sum_{r=1}^{m} \beta_{r} \alpha_{1}^{C_{r}}\right) \\
+ & P_{2}^{\prime i}\left(\sum_{r=1}^{m} \beta_{r} \alpha_{2}^{C_{r}}\right) \\
& P_{s}^{\prime i}\left(\sum_{r=1}^{m} \beta_{r} \alpha_{s}^{C_{r}}\right)
\end{aligned}
$$

It only needs to store the $\left(\sum_{r=1}^{m} \beta_{r} \alpha_{s}^{C_{r}}\right)$ values to use the predictor function with the scaled parameters. Table III shows these factor for the $T_{G A I T}$ and $T_{B A L}$ prediction function. Any new user condition could now be estimated as the product of their scaled spatiotemporal gait parameters (obtained at any moment of a walk using a time shifting window) by the factors described in table III.

We noticed in our tests that the model fitted volunteers affected by neurological disability $\left(R M S E\left(T_{\text {pred }}^{\text {gait }}\right)=\right.$ 1.51 and $\left.R M S E\left(T_{\text {pred }}^{b a l}\right)=1.97\right)$ better than the rest ( $\operatorname{RMSE}\left(T_{\text {pred }}^{\text {gait }}\right)=2.3$ and $\left.\operatorname{RMSE}\left(T_{\text {pred }}^{\text {bal }}\right)=3.4\right)$. This happens because all people in the neurological disability group were tested at the end of their rehabilitation process. At that point, they were already better and their physical condition allowed them to walk almost like healthy users $\left(T_{G A I T}=10.56 \pm 1.94, T_{B A L}=12.44 \pm 2.51\right)$. The other volunteers had poorer parameter values $\left(T_{G A I T}=7.05 \pm 2.6\right.$, $\left.T_{B A L}=10.84 \pm 4.07\right)$ and a much larger parameter variance, because they were at different states of recovery. Hence, the model provides a better fit for the neurological disability group in average.

\section{CONCLUSION AND FUTURE WORK}

In this paper we have presented a methodology to estimate user condition from gait parameters in challenged people

\begin{tabular}{|c|c|c|}
\hline Pmter. & $T_{\text {pred }}^{\text {gait }}$ & $T_{\text {pred }}^{\text {bal }}$ \\
\hline$C A D$ & 0.2707 & -1.7109 \\
\hline$S d T_{a v}$ & -0.3089 & -0.2942 \\
\hline$S d T_{s d}$ & -0.2852 & -0.7359 \\
\hline$S d L_{a v}$ & 0.3216 & 0.6483 \\
\hline$S d L_{s d}$ & 0.0642 & 0.5542 \\
\hline$S p T_{a v}^{L e f t}$ & -0.3256 & -0.3405 \\
\hline$S p T_{s d}^{L e f t}$ & -0.2625 & -0.1382 \\
\hline$S p T_{a v}^{R i g h t}$ & -0.2527 & -0.2129 \\
\hline$S p T_{s d}^{R i g h t}$ & -0.2878 & -1.0209 \\
\hline$S p L_{a v}^{L e f t}$ & 0.2109 & 0.9142 \\
\hline$S p L_{s d}^{\text {Left }}$ & 0.0606 & -0.0529 \\
\hline$S p L_{a v}^{R i g h t}$ & 0.2314 & 0.4968 \\
\hline$S p L_{s d}^{R i g h t}$ & 0.0626 & 0.1038 \\
\hline$\frac{s a}{W V}$ & 0.2338 & 1.4451 \\
\hline$U r S_{a v}^{L e f t}$ & 0.1128 & 0.5096 \\
\hline$U r S_{s d}^{L e f t}$ & -0.0545 & -0.7104 \\
\hline$U r S_{a v}^{\text {Right }}$ & 0.1202 & 0.6676 \\
\hline$U r S_{s d}^{R i g h t}$ & -0.0669 & -1.0508 \\
\hline
\end{tabular}

TABLE III

LOADING FACTORS

using a rollator equipped with force sensors and odometry. Unlike other works, our method does not require feedback from medical staff and estimation can be performed unsupervisedly, anytime and when users are performing any ADL. Our methodology imposes a single restriction to rollator users: they must support some weight on the platform. This is a loose restriction because, otherwise, they would not need the platform to walk. Our rollator can be used for long term monitoring and during the whole rehabilitation process. Our method is not as accurate as medical staff feedback. It has two errors in the response variable. The first one is introduced by the gait analysis algorithm, which has a small error associated to the spatial $3.53 \pm 0.0068 \%$, temporal $67.26 \pm 50.38 \mathrm{~ms}$ and support $0.98 \mathrm{~N}$ parameters. The second one is introduced by the regression, the RMSEP values are $T_{G A I T}=1.34$ and $T_{B A L}=2.31$. Nevertheless, results have proven that estimation is valid for a wide range of conditions: it has been validated with 19 volunteers presenting a variety of cognitive and physical disabilities.

Results have successfully proven that user condition prediction can be defined using the Tinetti Mobility Assessment, i.e. we could provide continuous assessment of $T_{G A I T}$ and $T_{B A L}$ to users and/or medical staff and we could use those values to adjust assistance on a need basis in the future. Hence, these results validate our proposal to estimate the user condition using only the spatiotemporal gait parameters on a rollator.

Future work will focus on extending this method to other mobility assessment tests as Berg Balance Scale [28] or balance evaluation system test [29], so we can explore the full potential of smart rollators as unsupervised evaluation tools. We will also use the user condition prediction in a collaborative control system [30] to continuously adapt the amount of help provided to users to their needs. 


\section{REFERENCES}

[1] D. o. E. United Nations and P. D. Social Affairs, "World population ageing 2015 report," 2015.

[2] S. Grammenos et al., "European comparative data on europe 2020 \& people with disabilities," 2013.

[3] S. W. Brose, D. J. Weber, B. A. Salatin, G. G. Grindle, H. Wang, J. J. Vazquez, and R. A. Cooper, "The role of assistive robotics in the lives of persons with disability," American Journal of Physical Medicine \& Rehabilitation, vol. 89, no. 6, pp. 509-521, 2010.

[4] M. M. Martins, C. P. Santos, A. Frizera-Neto, and R. Ceres, "Assistive mobility devices focusing on smart walkers: classification and review," Robotics and Autonomous Systems, vol. 60, no. 4, pp. 548-562, 2012.

[5] J. Shin, I. Steinmann, and B. Meyer, "Automatic speed control for smartwalker," in 8th Annual International Conference on Pervasive Technologies Related to Assistive Environments. ACM, 2015.

[6] C.-K. Lu, Y.-C. Huang, and C.-J. Lee, "Adaptive guidance system design for the assistive robotic walker," Neurocomputing, vol. 170, pp. 152-160, 2015.

[7] S. L. Grondin and Q. Li, "Intelligent control of a smart walker and its performance evaluation," in Rehabilitation Robotics (ICORR), 2013 IEEE International Conference on. IEEE, 2013, pp. 1-6.

[8] C.-H. Ko, K.-Y. Young, Y.-C. Huang, and S. K. Agrawal, "Active and passive control of walk-assist robot for outdoor guidance," Mechatronics, IEEE/ASME Transactions on, vol. 18, no. 3, pp. 1211-1220, 2013.

[9] C. Dune, P. Gorce, and J. Merlet, "Can smart rollators be used for gait monitoring and fall prevention?" in IEEE/RSJ Int. Conf. on Int. Rob. and Sys, 2012.

[10] U. Cortés, C. Barrué, F. Campana, R. Annicchiarico, and C. Caltagirone, "Towards an intelligent service to elders mobility using the i-walker." 2008.

[11] O. Chuy Jr, Y. Hirata, and K. Kosuge, "A new control approach for a robotic walking support system in adapting user characteristics," Systems, Man, and Cybernetics, Part C: Applications and Reviews, IEEE Transactions on, vol. 36, no. 6, pp. 725-733, 2006.

[12] W. M. Bortz II, "The disuse syndrome," Western Journal of Medicine, vol. 141 , no. 5 , p. $691,1984$.

[13] Y. Hirata, S. Komatsuda, and K. Kosuge, "Fall prevention control of passive intelligent walker based on human model," in Intelligent Robots and Systems, 2008. IROS 2008. IEEE/RSJ International Conference on. IEEE, 2008, pp. 1222-1228.

[14] M. E. Tinetti, "Performance-oriented assessment of mobility problems in elderly patients," Journal of the American Geriatrics Society, vol. 34, no. 2, pp. 119-126, 1986.

[15] R. Annicchiarico, C. Barrué, T. Benedico, F. Campana, U. Cortés, and A. Martínez-Velasco, "The i-walker: an intelligent pedestrian mobility aid." in ECAI, 2008, pp. 708-712.

[16] J. Ballesteros, C. Urdiales, A. B. Martinez, and M. Tirado, "Gait analysis for challenged users based on a rollator equipped with force sensors," in Intelligent Robots and Systems (IROS), 2015 IEEE/RSJ International Conference on. IEEE, 2015, pp. 5587-5592.

[17] J. W. Youdas, B. J. Kotajarvi, D. J. Padgett, and K. R. Kaufman, "Partial weight-bearing gait using conventional assistive devices," Archives of physical medicine and rehabilitation, vol. 86, no. 3, pp. 394-398, 2005.

[18] H. G. Kang and J. B. Dingwell, "Separating the effects of age and walking speed on gait variability," Gait \& posture, vol. 27, no. 4, pp. 572-577, 2008.

[19] D. A. Kegelmeyer, S. Parthasarathy, S. K. Kostyk, S. E. White, and A. D. Kloos, "Assistive devices alter gait patterns in parkinson disease: Advantages of the four-wheeled walker," Gait \& posture, vol. 38, no. 1, pp. 20-24, 2013.

[20] S. H. Peurala, E. B. Titianova, P. Mateev, K. Pitkänen, J. Sivenius, and I. M. Tarkka, "Gait characteristics after gait-oriented rehabilitation in chronic stroke," Restorative neurology and neuroscience, vol. 23, no. 2, pp. 57-65, 2005.

[21] A. L. Hof, "Scaling gait data to body size," Gait \& Posture, vol. 4, no. 3, pp. 222-223, 1996.

[22] D. Kleinbaum, L. Kupper, A. Nizam, and E. Rosenberg, Applied regression analysis and other multivariable methods. Nelson Education, 2013.

[23] T. Hastie, R. Tibshirani, J. Friedman, and J. Franklin, "The elements of statistical learning: data mining, inference and prediction," The Mathematical Intelligencer, vol. 27, no. 2, pp. 83-85, 2005.
[24] B.-H. Mevik, R. Wehrens, et al., "The pls package: principal component and partial least squares regression in r," Journal of Statistical Software, vol. 18, no. 2, pp. 1-24, 2007.

[25] M. Stone and R. J. Brooks, "Continuum regression: cross-validated sequentially constructed prediction embracing ordinary least squares, partial least squares and principal components regression," Journal of the Royal Statistical Society. Series B (Methodological), pp. 237-269, 1990.

[26] R. Kohavi et al., "A study of cross-validation and bootstrap for accuracy estimation and model selection," in Ijcai, vol. 14, no. 2, 1995, pp. 1137-1145.

[27] B.-H. Mevik and H. R. Cederkvist, "Mean squared error of prediction (msep) estimates for principal component regression (pcr) and partial least squares regression (plsr)," Journal of Chemometrics, vol. 18, no. 9, pp. 422-429, 2004.

[28] K. Berg, S. Wood-Dauphinee, and J. Williams, "The balance scale: reliability assessment with elderly residents and patients with an acute stroke." Scandinavian journal of rehabilitation medicine, vol. 27, no. 1, pp. 27-36, 1995 .

[29] F. B. Horak, D. M. Wrisley, and J. Frank, "The balance evaluation systems test (bestest) to differentiate balance deficits," Physical therapy, vol. 89, no. 5, pp. 484-498, 2009.

[30] C. Urdiales, M. Fernández-Carmona, J. Peula, R. Annicchiaricco, F. Sandoval, and C. Caltagirone, "Efficiency based modulation for wheelchair driving collaborative control," in Robotics and Automation (ICRA), 2010 IEEE International Conference on. IEEE, 2010, pp. 199-204. 